Geological Society, London, Special Publications Online First

\title{
Hydro-chemical modelling of in situ behaviour of bituminized radioactive waste in Boom Clay
}

N. Mokni, S. Olivella, E. Valcke, N. Bleyen, S. Smets, X. Li and X. Sillen

Geological Society, London, Special Publications, first published June 16, 2014; doi 10.1144/SP400.40

$\begin{array}{ll}\begin{array}{l}\text { Email alerting } \\ \text { service }\end{array} & \begin{array}{l}\text { click here to receive free e-mail alerts when } \\ \text { new articles cite this article }\end{array} \\ \begin{array}{l}\text { Permission } \\ \text { request }\end{array} & \begin{array}{l}\text { click here to seek permission to re-use all or } \\ \text { part of this article }\end{array} \\ \text { Subscribe } & \begin{array}{l}\text { click here to subscribe to Geological Society, } \\ \text { London, Special Publications or the Lyell } \\ \text { Collection }\end{array}\end{array}$

How to cite click here for further information about Online First and how to cite articles

Notes

(C) The Geological Society of London 2014 


\title{
Hydro-chemical modelling of in situ behaviour of bituminized radioactive waste in Boom Clay
}

\author{
N. MOKNI ${ }^{1 *}$, S. OLIVELLA ${ }^{1}$, E. VALCKE ${ }^{2}$, N. BLEYEN ${ }^{2}$, \\ S. SMETS ${ }^{2}$, X. LI $^{3} \&$ X. SILLEN ${ }^{4}$ \\ ${ }^{1}$ Department of Geotechnical Engineering and Geosciences, Universitat \\ Politècnica de Catalunya, Barcelona, Spain \\ ${ }^{2}$ Waste and Disposal Expert Group, The Belgian Nuclear Research \\ Centre $(S C K \bullet C E N)$, Boeretang 200, 2400 Mol, Belgium \\ ${ }^{3}$ EIG EURIDICE, Boeretang 200, 2400 Mol, Belgium \\ ${ }^{4}$ ONDRAF/NIRAS, Kunstlaan 14, 1210 Brussel, Belgium \\ *Corresponding author (e-mail: nadia.mokni@upc.edu)
}

\begin{abstract}
The hydro-chemical $(\mathrm{CH})$ interaction between swelling Eurobitum bituminized radioactive waste (BW) and Boom Clay was investigated to assess the feasibility of geological disposal for the long-term management of this waste. First, the long-term behaviour of BW in contact with water was studied. A CH formulation of chemically and hydraulically coupled flow processes in porous materials containing salt crystals is discussed. The formulation incorporates the strong dependence of the osmotic efficiency of the bitumen membrane on porosity and assumes the existence of high salt concentration gradients that are maintained for a long time and that influence the density and motion of the fluid. The impacts of temporal and spatial variations of key transport parameters (i.e. osmotic efficiency $(\sigma)$, intrinsic permeability $(k)$, diffusion, etc.) were investigated. Porosity was considered the basic variable. For BW porosity varies in time because of the water uptake and subsequent processes (i.e. dissolution of salt crystals, swelling of hydrating layers, compression of highly leached layers). New expressions of $\sigma$ and $k$ describing the dependence of these parameters on porosity are proposed. Several cases were analysed. The numerical analysis was proven to be able to furnish a satisfactory representation of the main observed patterns of the behaviour in terms of osmotic-induced swelling, leached mass of $\mathrm{NaNO}_{3}$ and progression of the hydration front when heterogeneous porosity and crystal distributions have been assumed. Second, the long-term behaviour of real Eurobitum drums in disposal conditions, and in particular its interaction with the surrounding clay, was investigated. Results of a $\mathrm{CH}$ analysis are presented.
\end{abstract}

Final disposal in deep geologically stable formations is considered worldwide as the preferred option for the long-term management of long-lived intermediate- and high-level radioactive waste. The disposal concepts proposed by many countries consist of a system of natural and engineered barriers to separate the waste from the biosphere. The Belgian Agency for the Management of Radioactive Waste and Fissile Materials (ONDRAF/NIRAS) envisages geological disposal of Eurobitum bituminized radioactive waste (BW) (ONDRAF/NIRAS 2009). Eurobitum is an intermediate-level longlived radioactive waste form that consists of $c$. $60 \mathrm{w} \%$ of hard bitumen Mexphalt R85/40 and $c$. $40 \mathrm{wt} \%$ of waste. The waste originates from the chemical reprocessing of spent nuclear fuel and from the cleaning of high-level waste storage tanks, and contains mainly $\mathrm{NaNO}_{3}$ and $\mathrm{CaSO}_{4}$. These salts are present with a concentration of, respectively, 20-30 and 4-6 wt\% of Eurobitum. In Belgium, the Boom Clay, which is a 30-35 million years old and c. $100 \mathrm{~m}$ thick marine sediment, is being studied as a potential host formation because of its favourable properties to limit and delay the migration of any leached radionuclides to the biosphere over extended periods of time. The current disposal concept foresees that several 2201 drums of Eurobitum would be grouped in a thick-walled cement-based secondary container, which in turn would be placed in concrete-lined disposal galleries that are excavated at mid-depth in the clay layer (Mariën et al. 2013). Only 80-90\% of the total volume of the drum would be filled with Eurobitum. The remaining voids between the drums would be backfilled with a cement-based material. The emplacement of Eurobitum BW in the Boom Clay is expected to result in a geomechanical and a physico-chemical perturbation of the clay (Valcke et al. 2010). The large amounts of dehydrated and hygroscopic salts in the $\mathrm{BW}$, with 


\section{N. MOKNI ET AL.}

bitumen acting as a highly efficient membrane, will provoke an osmosis-driven uptake of pore water, resulting in swelling of the BW and/or increased stress on the clay (i.e. a geomechanical perturbation of the clay). In addition, diffusion of large amounts of salts, mainly $\mathrm{NaNO}_{3}$, will result in physicochemical perturbation of the clay. After closure of the disposal gallery, two different phases can be distinguished (Valcke et al. 2010; Mariën et al. 2013). In the first, free swelling phase, an osmotically induced swelling of the BW will take place until all free volume is filled with swollen BW. Afterwards, the continued water uptake and swelling of the BW will induce an increasing stress on the clay. The resultant deformation of the clay creates additional space for the BW to swell, but compared with the free swelling phase, the swelling rate is limited by the increasing stress exerted by the clay. This second phase is the restricted swelling phase. The number of drums per gallery crosssection must be limited to prevent unacceptable geomechanical perturbations of the clay.

An experimental programme aiming at understanding the swelling and leaching behaviour of BW samples in contact with alkaline solutions is in progress at the Belgian Nuclear Research Centre SCK $\bullet$ CEN. The set of tests that has been performed consists of water uptake tests under restricted (i.e. constant stress and constant volume) and free swelling conditions (Valcke et al. 2010; Mariën et al. 2013). In a previous paper (Mokni et al. 2011) a hydro-chemical $(\mathrm{CH})$ formulation of chemically and hydraulically coupled flow processes in porous materials containing salt crystals was discussed. The formulation incorporates the strong dependence of the osmotic efficiency of the membrane on porosity, and assumes the existence of high concentration gradients that are maintained for a long time and that influence the density and motion of the fluid. The $\mathrm{CH}$ formulation was used to analyse the influence of osmosis on the swelling of Eurobitum BW owing to water uptake under laboratory conditions. The experimental results of pressure increase, swelling deformation and formation of low permeability compressed outer layers were reproduced successfully by Mokni et al. (2011) and Mariën et al. (2013). However, some differences were observed between the model results and the experimental observations in terms of progression of the hydration front and leached mass of $\mathrm{NaNO}_{3}$. Indeed, the modelled time to dissolve all salt crystals was shorter than observed in the experiments. According to the model, all crystals in the sample are dissolved after about 2000 days, while in reality only $12-21 \%$ of the initial $\mathrm{NaNO}_{3}$ content was leached after about 4 years of hydration and only the outer layers with a thickness of 1-2 mm were hydrated after this time period (Mariën et al.
2013). Mariën et al. (2013) stated that the difference is related to the simple relationship between porosity and membrane efficiency proposed by Mokni et al. (2011), which shows a too rapid decrease in efficiency with porosity increase (see Fig. 2 further in the text).

In order to better reproduce the experimental observations in terms of progression of the hydration front and leached mass of $\mathrm{NaNO}_{3}$, a new expression of the osmotic efficiency coefficient $(\sigma)$, including the dependence of $\sigma$ on porosity and on crystal volume fraction, is proposed in this paper. Because of the interdependency of most of the key transport functions (i.e. permeability, diffusion coefficient and osmotic efficiency) that control the magnitude of coupled fluxes (principally chemical osmosis and ultrafiltration), a new expression of the intrinsic permeability $(\kappa)$, including the dependence of $\kappa$ on porosity, is proposed in this paper. The water uptake tests under constant vertical stress performed on BW were modelled using the proposed expressions and several cases were analysed. A sensitivity analysis was performed in order to study the effect of the variation rates of $\sigma$ and $\kappa$ with porosity on swelling, leaching and progression of the hydration front of BW. The $\mathrm{CH}$ formulation was used to model the in situ behaviour of BW, to obtain insights into the kinetics of water uptake by BW, dissolution of the embedded $\mathrm{NaNO}_{3}$ crystals, solute leaching and maximum generated pressure under disposal conditions.

\section{Long-term swelling behaviour of BW: phenomenology and main processes observed in laboratory experiments}

A research programme involving the testing of the swelling behaviour of specimens of BW under constant volume and constant stress conditions was performed at the Belgian Nuclear Research Centre $\mathrm{SCK} \bullet \mathrm{CEN}$. The experimental results are reported in Valcke et al. (2010) and Mariën et al. (2013). Swelling under various constant stresses, osmosisinduced pressure in constant volume conditions and leached mass of $\mathrm{NaNO}_{3}$ were measured during the tests. After dismantling, the hydrated samples were characterized with micro-focus X-ray computer tomography $(\mu \mathrm{CT})$ and environmental scanning electron microscopy (ESEM). Modelling results of those tests were presented by Mokni et al. (2011); Mokni (2011) and Mariën et al. (2013). The phenomenology of the water uptake, swelling and salt leaching processes in restricted swelling conditions is now reasonably well understood (Valcke et al. 2010; Mokni et al. 2011; Mariën et al. 2013). The reported results and observations show that the uptake of water by and the subsequent swelling 
and/or pressure increase of BW (composed of $40 \mathrm{wt} \%$ of waste containing $28.5 \mathrm{wt} \%$ soluble salts $\mathrm{NaNO}_{3}$ ) are largely controlled by osmosis. The bitumen surrounding the soluble salts and other inorganic compounds behaves as a highly efficient semipermeable membrane, especially when tested under restricted swelling conditions. After almost four years of hydration of small BW samples (38 $\mathrm{mm}$ diameter, $10 \mathrm{~mm}$ thickness), osmosticinduced pressures of up to $20 \mathrm{MPa}$ and swelling deformations ranging between 10 and $20 \%$ are being measured in constant volume and constant stress water uptake tests, respectively. A major characteristic of the medium, which plays an important role in the process of water uptake, is the presence of salt crystals. In fact, the large amount of hygroscopic salt incorporated into the bitumen matrix will attract water from the surrounding reservoir, resulting in dissolution of the soluble crystals, diffusion of the dissolved salts and volume increase of the leaching BW. When the external applied stress is lower than the osmotic pressure, water is driven into the pores of the medium because of the gradient of concentration existing between the reservoir and the solution initially filling the pores. The pore water concentration will decrease until it reaches a value corresponding to an osmotic pressure equal to the external applied stress. The diffusion of $\mathrm{NaNO}_{3}$ out of the $\mathrm{BW}$, combined with the external stress and the osmosis-driven transport of water from the outermost pores with a lower $\mathrm{NaNO}_{3}$ concentration (higher water activity) towards deeper pores with a higher $\mathrm{NaNO}_{3}$ concentration (lower water activity), contributes to a local consolidation and the formation of a re-compressed, low-porosity layer at the outer boundary of the leached material (Fig. 1b). Compression of the outermost layers is considered here as a limiting or controlling process for the water uptake and the release of $\mathrm{NaNO}_{3}$. Valcke et al. (2010) characterized some leached samples with $\mu \mathrm{CT}$. This technique permitted the hydrated area of the leached sample to be visualized. Figure 1a shows the $\mu \mathrm{CT}$ image of samples S0 and S4 after 887 and 1472 days of hydration, respectively. The red parts represent the stainless steel filters. The thin green-yellow layers near the filters are the hydrated layers. The central part in red-yellow corresponds to the dry Eurobitum. For both samples, only a thin layer had been hydrated. The central part of the samples appeared to be not hydrated, which suggested that it would take several years before the hydration front reached the centre of the samples, and it would require even more time before all $\mathrm{NaNO}_{3}$ had dissolved. During that time, the sample was expected to continue to swell. In fact, size reduction of the outer pores induces a decrease in the permeability and the diffusion coefficient, and an increase in the osmotic efficiency in these compressed layers, while deeper in the sample these parameters vary in the opposite way. Therefore, the outer layers act as a highly efficient semipermeable membrane surrounding the swollen material with less efficient membrane properties. This maintains a very slow inflow of water towards the sample and outflow of the solute over a long time.

\section{Coupled CH formulation}

Mokni et al. $(2010 a, 2011)$ presented a $\mathrm{CH}$ formulation of chemically and hydraulically coupled flow processes in porous materials containing salt crystals, which incorporates the strong dependence of the efficiency of the membrane on porosity. The formulation assumes the existence of high concentration gradients that are maintained for a long time and that influence the density and motion of the fluid. The $\mathrm{CH}$ formulation is presented briefly below.

\section{Balance equations}

The medium is composed of three phases:

- solid phase - solid matrix and salt crystals;

- liquid phase $(l)$ - dissolved salt in water, dissolved air;

- gas phase $(g)$ - mixture of dry air and water vapour.

It also comprises four species:

- solid matrix $(m)$ - matrix embedding the crystals;

- salts - as crystal $(c)$ and as dissolved salt $(s)$ resulting from the dissolution of the crystals;

- water $(w)$ - as liquid or evaporated in the gas phase;

- air (a) - dry air as gas or dissolved in the liquid phase.

The total volume of the medium $\left(V_{t}\right)$ can be decomposed into the volume occupied by the crystals $\left(V_{c}\right)$, the pores (voids, $\left.V_{v}\right)$ and the bitumen $\left(V_{b}\right)$, that is, $V_{t}=V_{c}+V_{v}+V_{b}$. The volume of the pores varies as a consequence of dissolution of the crystals and may be occupied by water or air.

These volumes permit the volume fraction of the different components $\left(\phi_{c}\right.$ and $\left.\phi_{b}\right)$ and the volume fraction of pores, that is, the porosity $\left(\phi_{f}\right)$, to be defined. These variables are defined as:

$$
\begin{aligned}
\phi_{c} & =\frac{V_{c}}{V_{t}} ; \quad \phi_{f}=\frac{V_{v}}{V_{t}} ; \quad \phi_{b}=\frac{V_{b}}{V_{t}} \\
V_{t} & =V_{c}+V_{v}+V_{b} ; 1=\phi_{c}+\phi_{f}+\phi_{b}=\phi_{c}+\phi_{m} \\
\phi_{m} & =\phi_{f}+\phi_{b}
\end{aligned}
$$



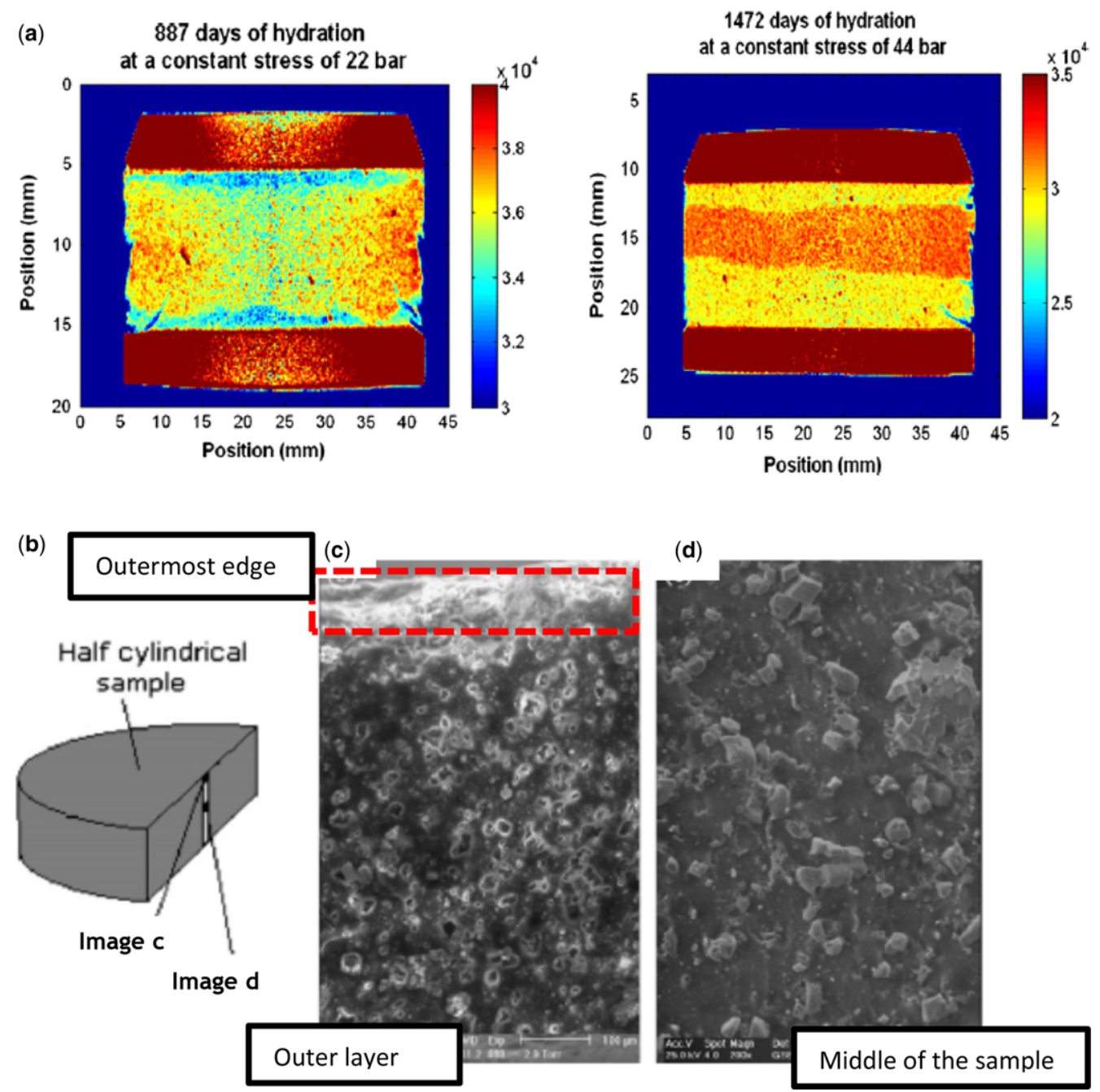

Fig. 1. (a) Micro-focus X-ray computer tomography ( $\mu \mathrm{CT}$ ) images of two Eurobitum samples: visualization of the hydration front in Eurobitum samples with $28.5 \mathrm{wt} \% \mathrm{NaNO}_{3}$ after 887 and 1472 days (Valcke et al. 2010). (b) ESEM photos of the leached layer of an Eurobitum sample (sample S0) after 887 days of contact with $0.1 \mathrm{M} \mathrm{KOH}$ in constant stress conditions (Valcke et al. 2010).

The sum of the porosity $\left(\phi_{f}\right)$ plus the volume fraction of the bitumen $\left(\phi_{b}\right)$ is defined as the volume fraction of the porous bitumen matrix $\phi_{m}$, that is, $\phi_{m}=\phi+\phi_{b}$. In this way, $1-\phi_{m}=$ $\phi_{c}$ is the volumetric fraction occupied by the crystals.

An initial porosity $\phi_{f_{0}}$ is assumed, corresponding to the volume initially occupied by the gas and water in contact with salt. As a consequence of the dissolution of the crystals, the pore volume available for the fluids $\left(\phi_{f}\right)$ will increase, resulting in a deformation of the sample.
To establish the balance equations, the compositional approach is adopted, consisting of balancing the species rather than the phases. The mass balance equations for water, dissolved salts, crystals and solid phase, including the coupled flows, namely osmotic flow and ultrafiltration, and the dissolution/precipitation of salts, are described in detail in Mokni et al. (2011). The formulation outlined below has been discretized in space and time in order to be used for numerical analysis and has been included in the computer code CODE_BRIGHT (Olivella et al. 1996). 


\section{Constitutive equations}

The following assumptions are made in the $\mathrm{CH}$ formulation:

- The medium constitutes a single soluble salt $\left(\mathrm{NaNO}_{3}\right)$ plus an inert solid phase.

- Multiphase flow including phase change is considered (dissolution/precipitation of the salt crystals).

- Only chemical and hydraulic gradients are considered.

- The medium is assumed not to be saturated with water.

- Binary diffusion of salt and water at high concentration is assumed.

- Permeability, diffusion and osmotic efficiency coefficients are considered to be variable with porosity.

The constitutive equations that relate the massaverage flow of fluid and the mass flow of solute to chemical and pressure gradients are as follows (Mokni et al. 2011):

(1) Flux of liquid phase ( $\left.\mathbf{J}_{l}\right)$ induced by both pressure and concentration gradients:

$$
\begin{aligned}
& \mathbf{J}_{l}=-\frac{\mathbf{k} k_{r l}}{\mu}\left(\nabla p+\rho_{l} \mathbf{g} \nabla z\right)+\frac{\alpha_{s}}{\mu} \mathbf{k} \sigma \nabla\left(w_{l}^{s}\right) ; \\
& \alpha_{s}=\frac{k_{r l} R T \rho_{l}}{M_{s}}
\end{aligned}
$$

This is an advective flux in the sense that it transports both the solute and the water.

(2) Nonadvective flux of solute:

$$
\begin{aligned}
\mathbf{J}_{l}^{s} & =-\sigma w_{l}^{s} \rho_{l} \mathbf{J}_{l}-D \rho_{l}(1-\sigma)\left(1+\gamma w_{l}^{s}\right) \nabla w_{l}^{s} ; \\
\gamma & =\frac{1}{\rho_{l}} \frac{\partial \rho_{l}}{\partial w_{l}^{s}}
\end{aligned}
$$

(3) Nonadvective flux of water including ultrafiltration is written as:

$$
\begin{aligned}
\mathbf{J}_{l}^{w} & =\frac{\beta}{\alpha} \sigma w_{l}^{s} \rho_{l} \mathbf{J}_{l}-D \rho_{l}(1-\sigma)\left(1-\gamma w_{l}^{w}\right) \nabla w_{l}^{w} \\
\alpha & =1+\frac{1}{\rho_{l}} \frac{\partial \rho_{l}}{\partial w_{l}^{s}} w_{l}^{s}=1+\gamma w_{l}^{s} \\
\beta & =1+\frac{1}{\rho_{l}} \frac{\partial \rho_{l}}{\partial w_{l}^{w}} w_{l}^{w}=1-\gamma w_{l}^{w}
\end{aligned}
$$

where $\mathbf{k}\left(\mathrm{m}^{2}\right)$ denotes the intrinsic permeability tensor, $k_{r l}$ the relative permeability of the liquid, $\mathbf{g}$ the gravity vector, $\mu$ (MPa s) the dynamic viscosity, $p(\mathrm{MPa})$ the hydraulic pressure, $w_{l}^{s}$ the mass fraction of solute in the liquid phase, $w_{l}^{w}$ the mass fraction of water in the liquid phase, $R\left(\mathrm{~J}(\mathrm{~mol} \mathrm{~K})^{-1}\right)$ the gas constant, $T(\mathrm{~K})$, the temperature, $\rho_{l}$ $\left(\mathrm{kg} \mathrm{m}^{-3}\right)$ the liquid density, $M_{s}\left(\mathrm{~kg} \mathrm{~mol}^{-1}\right)$ the solute molar mass, $\sigma$ the osmotic efficiency coefficient, and $D\left(\mathrm{~m}^{2} \mathrm{~s}^{-1}\right)$ the diffusion coefficient.

The total fluxes of water and solutes in the liquid phase are expressed as:

$$
\begin{aligned}
\mathbf{j}_{l}^{w}= & -w_{l}^{w} \rho_{l} \frac{\mathbf{k}}{\mu} \nabla p+D(1-\sigma) \rho_{l}\left(1-\gamma w_{l}^{w}\right) \nabla w_{l}^{s} \\
& +\frac{\mathbf{k} \sigma}{\mu}\left(w_{l}^{w} \rho_{l}\right) \nabla \pi+\frac{\mathbf{k} \boldsymbol{\sigma}^{2}}{\mu}\left(\frac{\beta}{\alpha} w_{l}^{w} \rho_{l}\right) \nabla \pi \\
& -\frac{\mathbf{k} \sigma}{\mu}\left(\frac{\beta}{\alpha} w_{l}^{w} \rho_{l}\right) \nabla p+w_{l}^{w} \rho_{l} \phi_{f} \frac{d \mathbf{u}}{d t} \\
\mathbf{j}_{l}^{s}= & -w_{l}^{s} \rho_{l} \frac{\mathbf{k}}{\mu} \nabla p-D(1-\sigma) \rho_{l}\left(1+\gamma w_{l}^{s}\right) \nabla w_{l}^{s} \\
& +\frac{\mathbf{k} \sigma}{\mu}\left(w_{l}^{s} \rho_{l}\right) \nabla p-\frac{\mathbf{k} \sigma^{2}}{\mu}\left(w_{l}^{s} \rho_{l}\right) \nabla \pi \\
& +\frac{\mathbf{k} \sigma}{\mu}\left(w_{l}^{s} \rho_{l}\right) \nabla \pi+w_{l}^{s} \rho_{l} \phi_{f} \frac{d \mathbf{u}}{d t}
\end{aligned}
$$

The first and second terms in equations (5a) and (5b) represent Darcy's and Fick's laws, respectively. The solute diffusion law is dependent upon the osmotic efficiency coefficient $(\sigma)$. This is an additional coupling effect included in order to model diffusion for semipermeable membrane materials. For the total flux of water (equation 5a) the third term represents chemical osmosis. The fourth and the fifth terms are coupled fluxes representing respectively the flow of water induced by an osmotic $(\nabla \pi)$ and a hydraulic $(\nabla p)$ pressure gradient. For the total flux of the solute, the coupled fluxes terms include ultrafiltration (third term). The solute flow is also driven by an osmotic pressure gradient (fourth and fifth terms in equation $5 b)$. The sixth terms in both equations describe the advective flux owing to solid motion.

\section{Constitutive relationships}

Important parameters that affect the water uptake and salt release rates and hydration front are the bitumen membrane efficiency, the permeability of the BW, and the diffusion coefficient of dissolved salt in the BW. These parameters depend on the porosity of the BW, which varies in time because of the water uptake and subsequent processes, that is, dissolution of salt crystals, swelling of hydrating layers and compression of highly leached layers.

The diffusivity of solutes and water $\left(m^{2} s^{-1}\right)$. The diffusion coefficient controls the diffusion of dissolved salt and water. The parameter $D$ is considered as an effective diffusion coefficient equal to $D=\tau \phi_{f} D_{0}$ where $\tau$ is the tortuosity and $D_{0}$ is 


\section{N. MOKNI ET AL.}

a Fickian diffusion coefficient for a solute in free water. The effective diffusivity for semipermeable membranes is defined as $D^{*}=(1-\sigma) D$. Therefore, for media presenting ideal membrane properties, the diffusion of the solute and water is completely hindered.

The osmotic efficiency $\sigma$. The osmotic efficiency coefficient controls the flow of water and solutes owing to osmotic effects. This parameter describes the nonideality of membrane behaviour. It ranges from 1 for an ideal membrane to 0 for porous media having no membrane properties. For clays, it has been shown that the efficiency of the clay membrane depends on several factors such as cation exchange capacity, solute concentration or porosity. For a composite porous medium such as $\mathrm{BW}$, composed of a porous bituminized matrix embedding crystals, Mokni et al. (2011) proposed a simple relationship that describes the decrease of $\sigma$ when porosity increases:

$$
\sigma=\sigma_{0}\left(\frac{\phi_{0}}{\phi_{f}}\right) ; \quad \phi_{0} \leq \phi_{f}
$$

This equation shows a rapid decrease in osmotic efficiency with porosity increase (Fig. 2a).

(a)
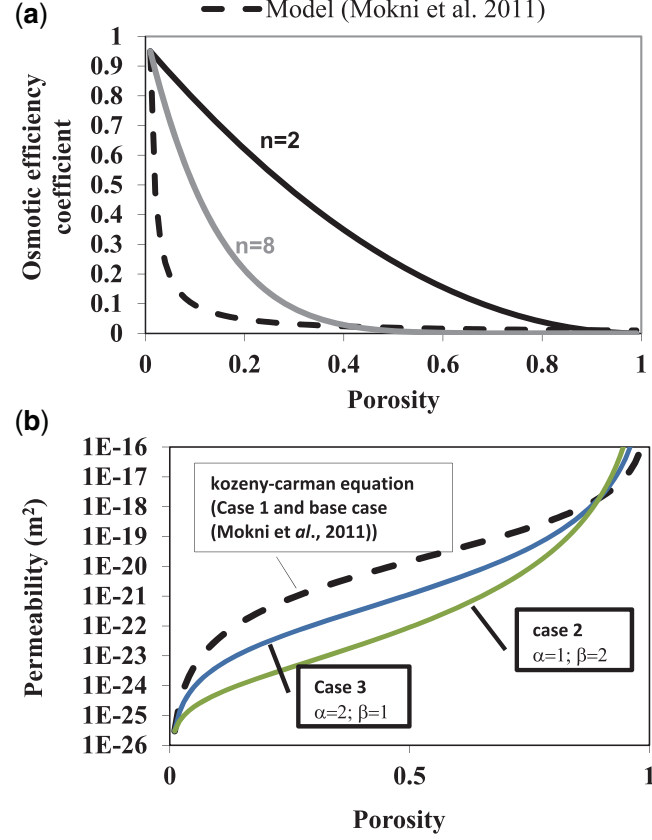

Fig. 2. Evolution of (a) osmotic efficiency coefficient for different values of $n$ in equation (6b) and for previous function used in Mokni et al. (2011) and (b) intrinsic permeability used in the different analysed cases and Kozeny-Carman function used in Mokni et al. (2011).
In the present paper, the osmotic efficiency coefficient is assumed to be dependent on porosity and on crystal content. The latter dependency has been reported by Valcke et al. (2010), who mentioned the existence of a threshold salt content at which BW loses its membrane properties. Indeed, for high crystal contents, the bitumen membrane around the salt crystals becomes too thin to induce any osmotically driven swelling.

The osmotic efficiency coefficient $\sigma$ is assumed to be a nonlinear function of the porosity $\left(\phi_{f}\right)$ and of the crystal volume fraction $\left(\phi_{c}\right)$ :

$$
\sigma=\sigma_{0}\left(\frac{1-\phi_{f}}{1-\phi_{0}}\right)^{n}\left(1-\phi_{c}-\phi_{f}\right) ; \quad \phi_{0} \leq \phi_{f}
$$

where $\sigma_{0}$ is the reference osmotic efficiency for the reference porosity $\phi_{0}$, and $n$ is a material parameter that controls the rate of decrease of $\sigma$ with increase of $\phi_{f}$ and $\phi_{c}$. Figure 2a shows the evolution of the osmotic efficiency coefficient as a function of porosity for several values of $n$. The choice of this expression for the membrane efficiency is based on experimental observations. Indeed, there is experimental evidence that, even when the layers are partially leached, the membrane efficiency is still very high (Valcke et al. 2010).

The intrinsic permeability $\mathrm{k}$. The intrinsic permeability controls the flow of water owing to hydraulic and osmotic pressure gradients. It also controls the flow of solutes by advection. Mokni et al. (2011) used a Kozeny-Carman-type equation (Fig. 2b) to describe the evolution of permeability with porosity expressed as:

$$
k=k_{0} \frac{\phi_{f}^{3}}{\left(1-\phi_{f}\right)^{2}} \frac{\left(1-\phi_{0}\right)^{2}}{\phi_{0}^{3}}
$$

This equation is useful for soil materials. Nevertheless, for a composite porous media such as $\mathrm{BW}$, experiencing a structure evolution induced by dissolution and swelling, an expression that is specifically applicable to that particular material is needed. Mokni et al. (2011) performed a sensitivity analysis on the effect of the initial values of intrinsic permeability, osmotic efficiency, and diffusion coefficients on the magnitude and duration of swelling deformation of BW. It was demonstrated that these three parameters, dependent on porosity, evolve in a coupled way. Indeed, a material having a high value of permeability and diffusion has a low value of the efficiency coefficient (less efficient semi permeable membrane).

In this paper, in order to investigate the effect of the rate of change of permeability with porosity, a generalized form of equation (7a) is proposed for BW. 


$$
k=k_{0} \frac{\phi_{f}^{\alpha}}{\left(1-\phi_{f}\right)^{2 \beta+2}} \frac{\left(1-\phi_{0}\right)^{2 \beta+2}}{\phi_{0}^{\alpha}}
$$

where $k_{0}$ is the reference permeability at the reference porosity $\phi_{0}$, and $\alpha$ and $\beta$ are material parameters between 1 and 2 that control the rate of increase of $k$ with $\phi_{f}$. Figure $2 \mathrm{~b}$ shows the evolution of the intrinsic permeability as a function of porosity for several values of $\alpha$ and $\beta$.

\section{Sensitivity analysis: effect of the rate of variation of $\sigma$ and $k$ with porosity on swelling, leached mass of $\mathrm{NaNO}_{3}$ and on leached thickness of BW}

In this section, results from the modelling work of the water uptake tests under constant stress conditions described in Valcke et al. (2010) using the proposed expressions of $\sigma$ and $k$ (equations $6 \mathrm{~b} \&$ $7 b)$ are presented. Several cases are analysed considering different values of $n, \alpha$ and $\beta$, parameters controlling the rate of change of osmotic efficiency and intrinsic permeability with porosity, swelling, leached mass of $\mathrm{NaNO}_{3}$ and leached thickness.

For the experimental aspects of these water uptake tests, the reader is referred to Valcke et al. (2010). Specifically for this paper, it is important to mention that, prior to contact with water, the samples in the water uptake cells were submitted to repeated compression-decompression steps ('compressibility test') to determine the mechanical properties of BW. Mokni et al. (2008, 2010a, b) proposed an elasto-viscoplastic constitutive model to simulate these compression tests in oedometric conditions. The $\mathrm{CH}$ formulation, combined with the elasto-viscoplastic mechanical constitutive model (Mokni et al. 2010b) was used to simulate the water uptake tests. The result of the different analyses performed will be compared with the result of the Base case described in Mokni et al. (2011).

\section{Model domain, boundary conditions and (initial) parameter values}

The model domain consists of two regions (Fig. 3). One region corresponds to a reservoir representing a filter that is saturated with a low $\mathrm{NaNO}_{3}$ concentration solution. The initial $\mathrm{NaNO}_{3}$ mass fraction for the reservoir is $w=0.001 \mathrm{~kg} \mathrm{NaNO}_{3} \mathrm{~kg}^{-1}$ solution $(c .0 .01 \mathrm{M})$. Within the reservoir, the permeability is set to high values $\left(k=10^{-16} \mathrm{~m}^{2}\right)$. The other region corresponds to the $\mathrm{BW}$ containing $\mathrm{NaNO}_{3}$ crystals occupying $16 \%$ of the total volume of the $\mathrm{BW}$ (corresponding to $28 \mathrm{wt} \%$ ).

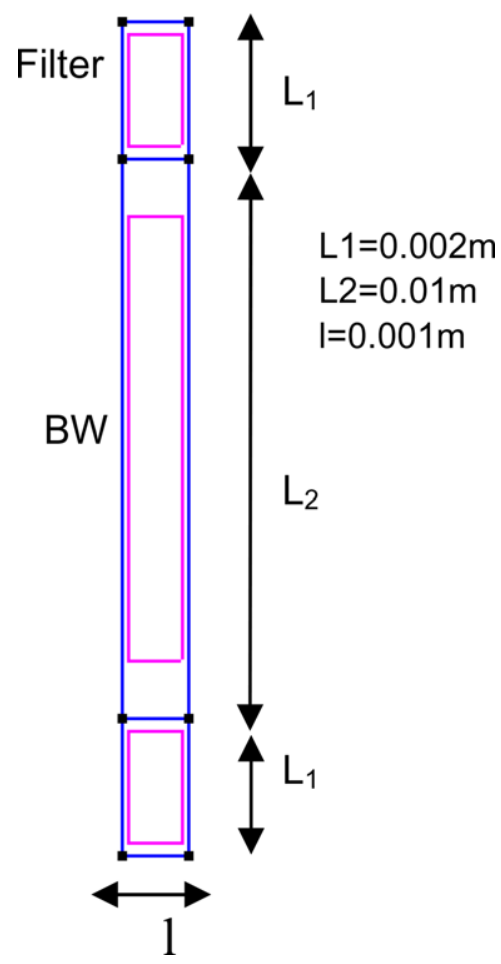

Fig. 3. Schematic representation of the model domain.

Pure water inflow, brine outflow and salt diffusion through the reservoir are allowed at the upper and lower sides of the sample. The medium is considered to be saturated $\left(S_{\mathrm{r}}=1\right)$ with $\mathrm{NaNO}_{3}$ brine (initial mass fraction $w=0.47 \mathrm{~kg} \mathrm{NaNO}_{3} \mathrm{~kg}^{-1}$ solution (c. $7.46 \mathrm{M})$ ).

The different cases were simulated under a constant vertical stress. Along the vertical boundaries of the domain, horizontal displacements are restricted to represent the oedometric conditions. Material properties are summarized in Table 1. The initial values for permeability, osmotic efficiency and diffusion coefficient are the same reference values used by Mokni et al. (2011) and Marien et al. (2013). The mechanical parameters were derived from the calibration of oedometer compressibility tests that are presented in Mokni et al. (2010b).

\section{Effect of $\alpha$ and $\beta$ on swelling and leached mass of $\mathrm{NaNO}_{3}$}

Table 2 summarizes the different cases analysed. The transport functions (permeability and osmotic efficiency) used for the different cases are displayed in Figure 2b. 
Table 1. Material properties

\begin{tabular}{llll}
\hline Parameter (initial values) & Symbol & Value \\
\hline Crystal volume fraction & $\phi_{c_{0}}$ & & 0.16 \\
Porosity & $\phi_{f_{0}}$ & & 0.01 \\
Intrinsic permeability & $k_{0}\left(\mathrm{~m}^{2}\right)$ & $\mathrm{BW}$ & $3 \times 10^{-26}$ \\
& $k\left(\mathrm{~m}^{2}\right)$ & Filter & $10^{-16}$ \\
Efficiency coefficient & $\sigma_{0}$ & & 0.95 \\
Diffusion coefficient & $D^{*}\left(\mathrm{~m}^{2} \mathrm{~s}^{-1}\right)$ & & $1.6 \times 10^{-16}$ \\
Dissolution rate constant & $\kappa\left(\mathrm{kg} \mathrm{s}^{-1} \mathrm{~m}^{-3}\right)$ & $\mathrm{BW}$ & 0.47 \\
Solute mass fraction & $w_{l}^{s}\left(\mathrm{~kg} \mathrm{NaNO}_{3} \mathrm{~kg}^{-1}\right.$ solution) & Filter & 0.001 \\
& & & \\
\hline
\end{tabular}

Initial values of transport parameters used for the different cases.

BW, Eurobitum bituminized radioactive waste.

Table 2. Transport functions used for the different cases

\begin{tabular}{lll}
\hline Analysed cases & \multicolumn{1}{c}{ Constitutive relationships } \\
\hline Case 1* & $k=k_{0} \frac{\phi_{f}^{3}}{\left(1-\phi_{f}\right)^{2}} \frac{\left(1-\phi_{0}\right)^{2}}{\phi_{0}{ }^{3}} ; \quad \sigma=\sigma_{0}\left(\frac{1-\phi_{f}}{1-\phi_{0}}\right)^{n} ; n=2,3,5$ \\
Case 2 & $k=k_{0} \frac{\phi_{f}}{\left(1-\phi_{f}\right)^{6}} \frac{\left(1-\phi_{0}\right)^{6}}{\phi_{0}} ; \quad \sigma=\sigma_{0}\left(\frac{1-\phi_{f}}{1-\phi_{0}}\right)^{n} ; n=2,3,8$ \\
Case 3 & $k=k_{0} \frac{\phi_{f}{ }^{2}}{\left(1-\phi_{f}\right)^{4}} \frac{\left(1-\phi_{0}\right)^{4}}{\phi_{0}{ }^{2}} ; \quad \sigma=\sigma_{0}\left(\frac{1-\phi_{f}}{1-\phi_{0}}\right)^{2} ; n=2$ \\
\hline
\end{tabular}

*Kozeny-Carman function used in Mokni et al. (2011).

Case 1. In this case the Kozeny-Carman type permeability function (equation 7a) used by Mokni et al. (2011) is maintained, while for the osmotic efficiency coefficient the new expression (equation 6b) that describes the dependency of $\sigma$ on $\phi_{f}$ and $\phi_{c}$ is used. Several values of $n$ are considered. The evolution of volumetric deformation (i.e. swelling) is displayed in Figure 4. The plot shows

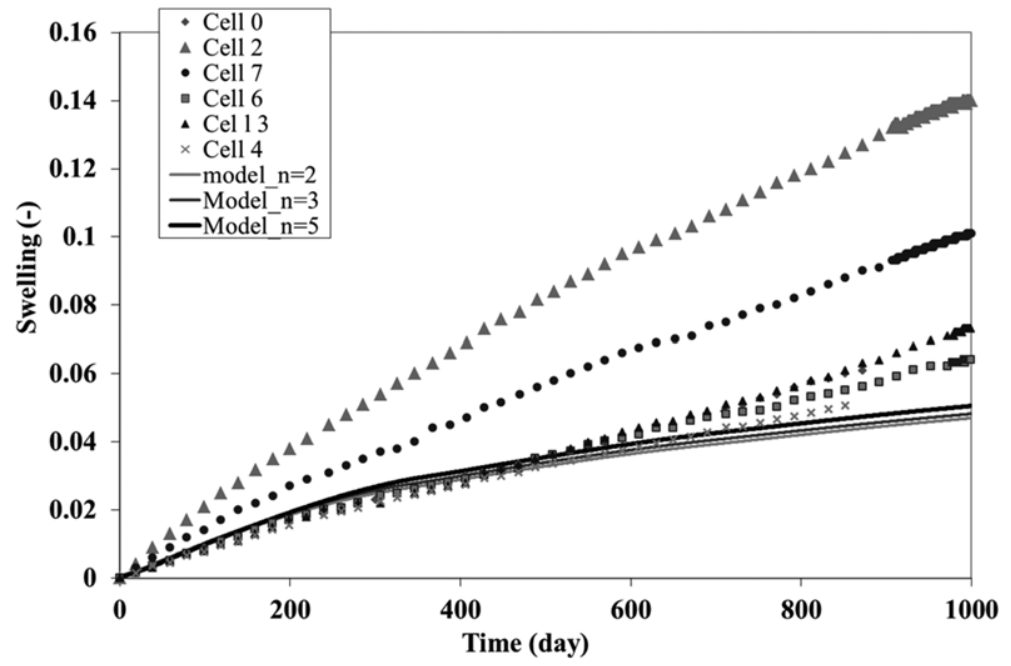

Fig. 4. Case 1: swelling (or volumetric deformation) for different values of $n$ (equation 6b). 


\section{MODELLING BEHAVIOUR OF BW IN BOOM CLAY}

comparisons between the experimental data and the numerical simulations (at constant vertical applied stress, 2.2 MPa). It is observed that the model predicts lower swelling rates in comparison to the experimental results. Indeed, in the $\mathrm{CH}$ formulation, water uptake, swelling and leaching are considered to be the result of several transient fully coupled processes (diffusion, advection, dissolution, involving a low permeability material with evolving thickness and properties) that are strongly nonlinear. The overall time-dependence of the swelling and leaching rates and rate of progression of the hydration front depend on the relative contribution of all these processes and on their time constants. In fact, the variation of the intrinsic permeability and osmotic coefficient plays an important role in the coupled migration of water and solute. While the direct advective fluxes are proportional to $k$, the coupled fluxes are proportional to the product $k \sigma$ (equation 5). It is convenient to define at this point the following transport functions: $k \pi / \mu$, $k \pi \sigma / \mu$ and $k \pi \sigma^{2} / \mu$. These functions will be compared with the diffusion coefficient $D^{*}$ to identify the major processes that control the transport of water and solute. Figure 5 shows the evolution with porosity of the above-defined functions together with the diffusion coefficient $D^{*}$ for case 1 (Fig. 5a) and for the base case presented by Mokni et al. (2011) (Fig. 5b). The plots indicate that, for case 1 , the transport functions controlling the swelling and leaching rates are significantly different from the base case. Indeed, for case 1 the transport functions are similar and evolve with porosity at similar rates. In addition, higher values and
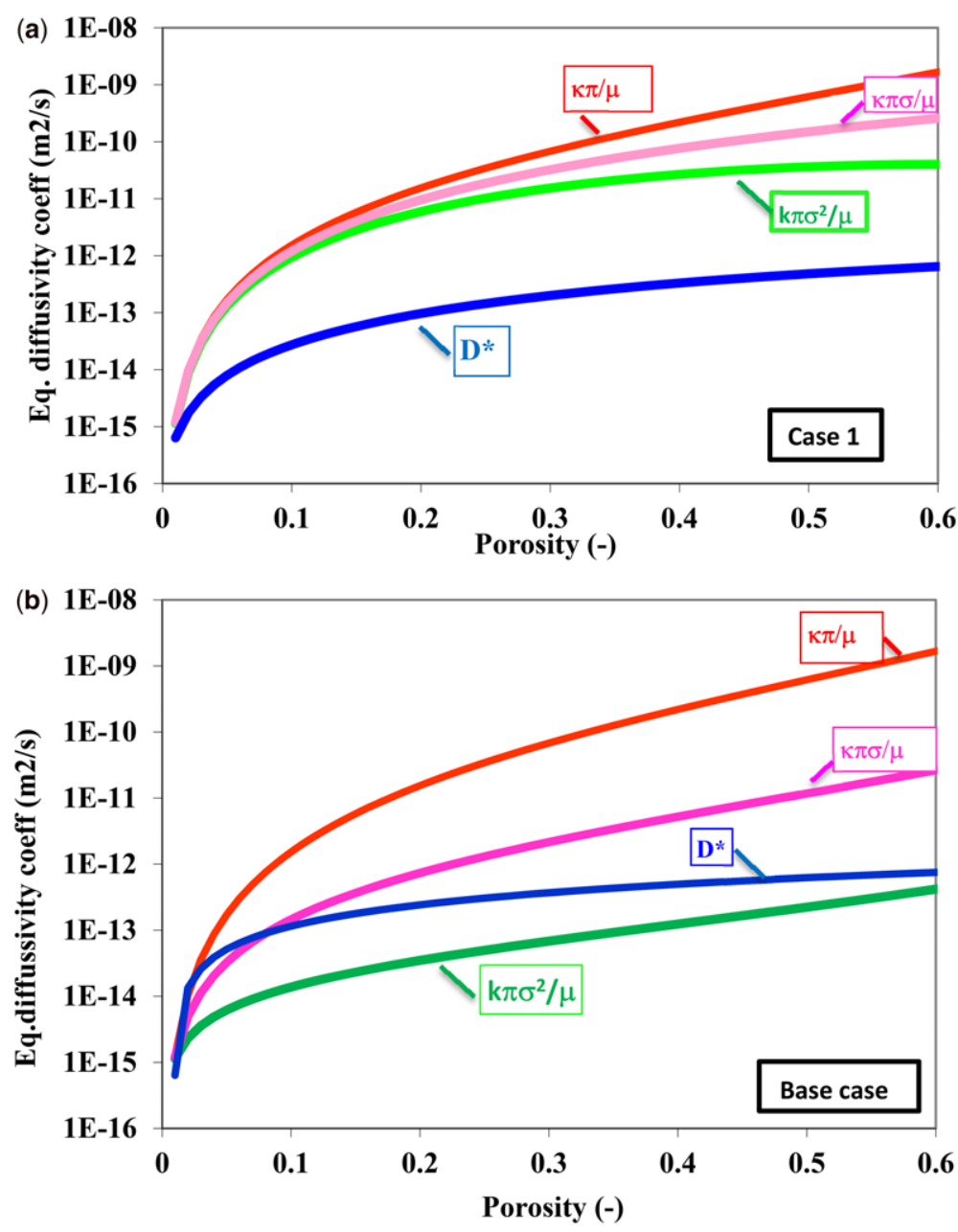

Fig. 5. Variation with porosity of the transport functions $k \pi / \mu, k \pi \sigma / \mu, k \pi \sigma^{2} / \mu$ and $D^{*}$ for (a) case 1 and (b) base case (Mokni et al. 2011). 


\section{N. MOKNI ET AL}

evolution rate are observed for the transport function $k \pi \sigma^{2} / \mu$ in comparison to the base case. As a result there is rapid leaching of the outermost layers of the BW sample and a rapid decrease in $\mathrm{NaNO}_{3}$ concentration and therefore in osmotic pressure in these layers owing to the higher water and solute flow rates. The transport of $\mathrm{NaNO}_{3}$ solutes out of the BW, combined with the external stress and the osmosis-driven transport of water from the outermost pores with a lower $\mathrm{NaNO}_{3}$ concentration (higher water activity) towards deeper pores with a higher $\mathrm{NaNO}_{3}$ concentration (lower water activity), contributes to a local consolidation of the hydrated layers. Swelling of the deeper layer is then counteracted by compression of the leached layers leading to a fast levelling off of the leaching and swelling rates.
Case 2. In this case the new permeability (equation 7b) and osmotic efficiency (equation 6b) functions are used (Table 2). The used permeability function predicts a slower increase of the permeability with increasing porosity compared with the base case (Fig. 2b). Several values of the parameter $n$, which controls the rate of decrease of the osmotic efficiency coefficient with porosity, are considered. The same initial values of the transport function as for the previous case have been used (Table 1). The evolution of volumetric deformation is displayed in Figure 6. Experimental and numerical results concerning the sodium nitrate release are shown in Figure 7. In this case the model reproduces well the swelling and leaching rates. Several observations can be made. First, it is seen that, after more than 4 years of hydration, the samples

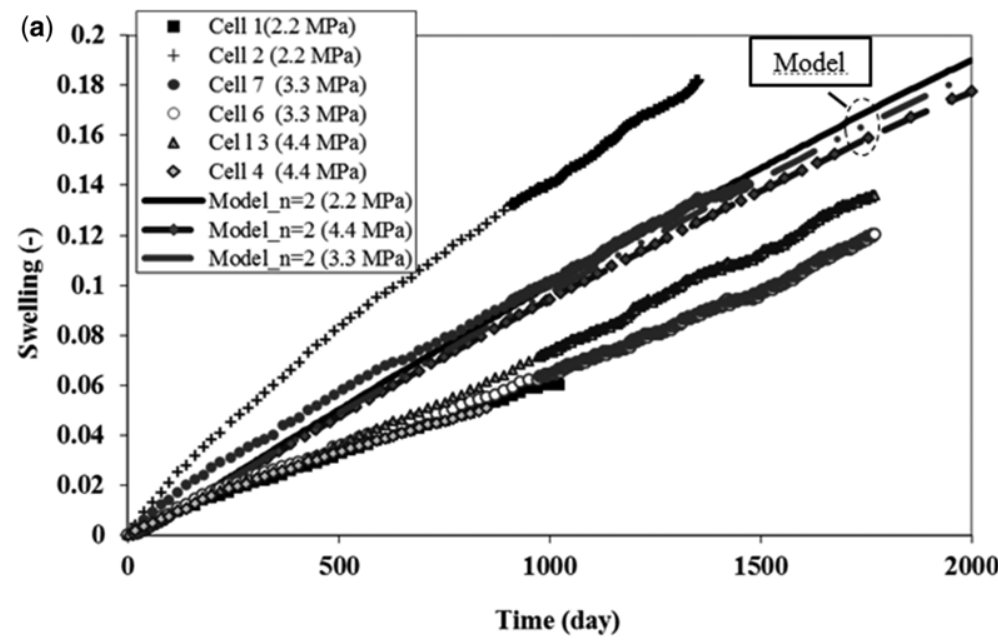

(b)

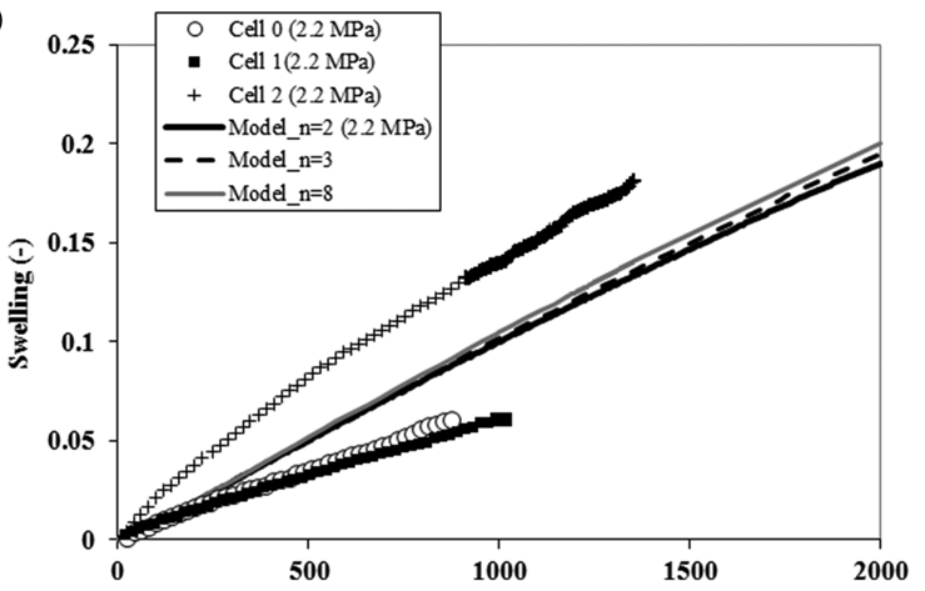

Fig. 6. Case 2: swelling (or volumetric deformation) for (a) different applied stresses and (b) different values of $n$. Experimental results and model predictions. 


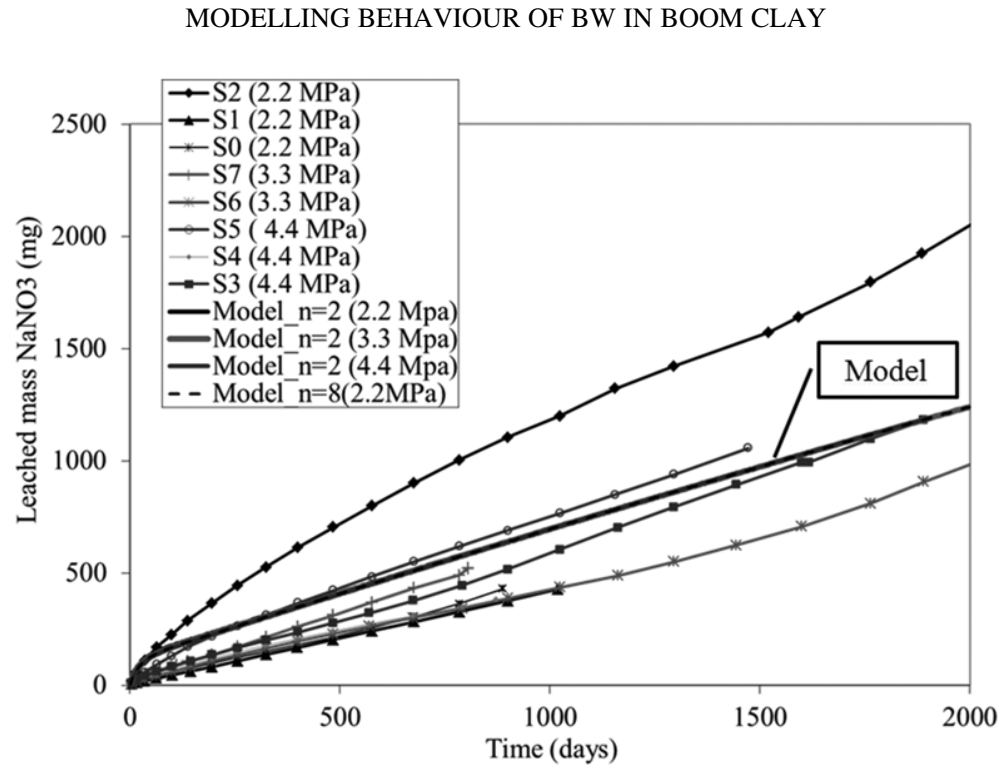

Fig. 7. Case 2: leached mass of $\mathrm{NaNO}_{3}$. Experimental results and model predictions.

continued to swell with no tendency to level off. Both modelling and experiments were performed under various vertical applied stresses $(2.2,3.3$, and 4.4 MPa). For this range of stresses, the effect on swelling and leaching rates is moderate according to the model. The effect of stress level on swelling was discussed by Mokni et al. (2011) in regard to the maximum swelling pressure achievable taking into account the high osmotic pressure associated with saturated sodium nitrate pore solution. Second, no significant effect of the variation of the value of $n$ is observed on the swelling and leaching rates (Figs $6 \mathrm{~b} \& 7$ ). Figure 8 shows the evolution with porosity of the above-defined transport functions together with the diffusion coefficient $D^{*}$ for several values of the parameter $n$. The plot shows that, in both cases (i.e. cases of $n=8$ and $n=2$ ) for low porosity, diffusion is the dominant process for the transport of water and solute. As porosity increases, permeability increases and the direct and coupled advective fluxes become the dominant processes. For both cases, the transport parameters controlling the swelling and leaching rates are significantly different at high porosity values, while more similar values are observed for low porosities. The fact that no effect of $n$ on the swelling

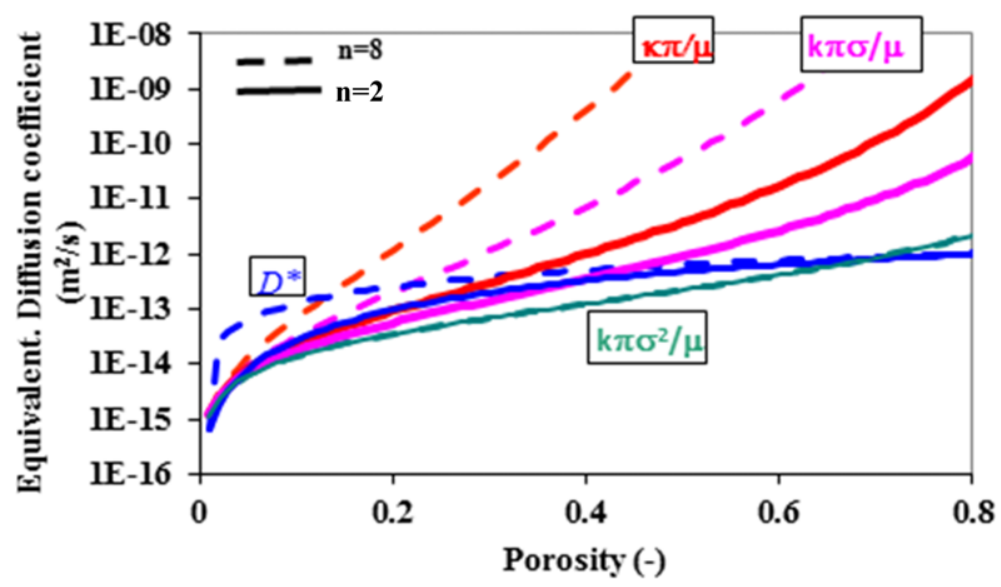

Fig. 8. Case 2: variation with porosity of the transport functions $k \pi / \mu, k \pi \sigma / \mu$ and $k \pi \sigma^{2} / \mu$ and $D^{*}$, which control the magnitude of the direct and coupled fluxes of water and solute (for a given osmotic and hydraulic pressure gradients; $\pi=42 \mathrm{MPa}$ and $\mu=10^{-9} \mathrm{MPa}$ ). 


\section{N. MOKNI ET AL.}

and leaching rates is observed demonstrates that both are controlled by the fluxes in the outermost low porosity layer. Compression of the outermost layers is then a limiting or controlling process for the water uptake and the release of $\mathrm{NaNO}_{3}$.

Case 3. In this case, the rate of increase of permeability with porosity is higher than in case 2 but lower than in case 1 (Fig. 2a; Table 2). The same material parameters as for the previous cases have been used (Table 1). Modelling results of swelling and $\mathrm{NaNO}_{3}$ leaching of the samples in the constant stress test $\left(\sigma_{\mathrm{v}}=2.2 \mathrm{MPa}\right)$ leads to lower swelling and leaching rates in comparison to the experimental results (Fig. 9). In fact, in this case the outermost layers are predicted to leach quite rapidly owing to the higher water and solute flow rates inducing a fast decrease in $\mathrm{NaNO}_{3}$ concentration and in osmotic pressure in these layers. The osmotic pressure would then rapidly approach the value of the externally applied stress, which would lead to compression of the hydrated layers.

\section{Effect of $\alpha$ and $\beta$ on leached thickness of $B W$ and hydration front}

The progression of the water front is the result of the relative contribution of several transient processes (diffusion, advection and dissolution) involving a low porosity and low permeability material with evolving thickness and properties and that are strongly coupled. Valcke et al. (2010) and Mariën et al. (2013) observed that almost no pores can be seen on ESEM images of the dry BW. Indeed,

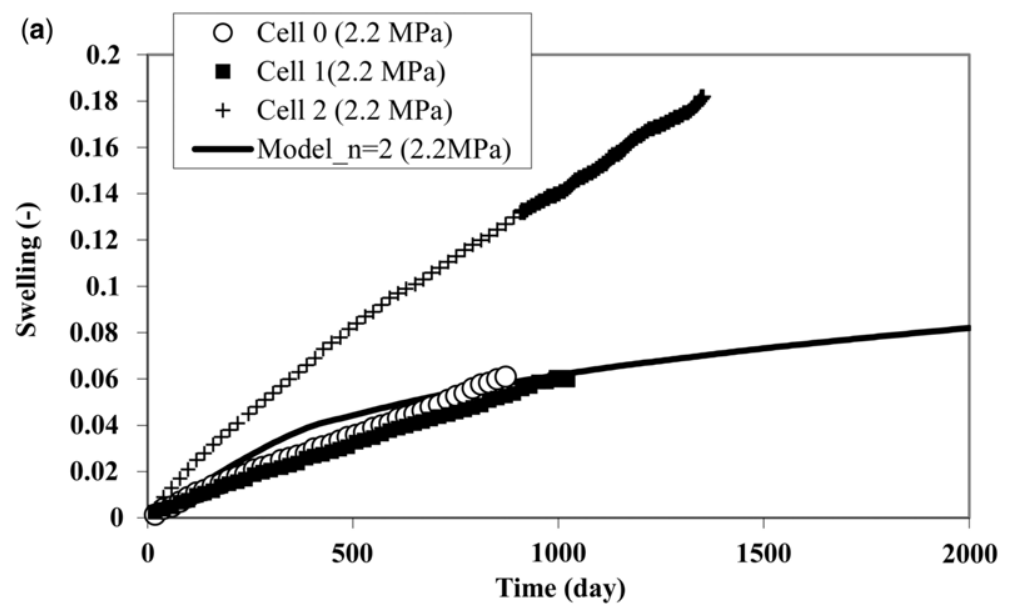

(b)

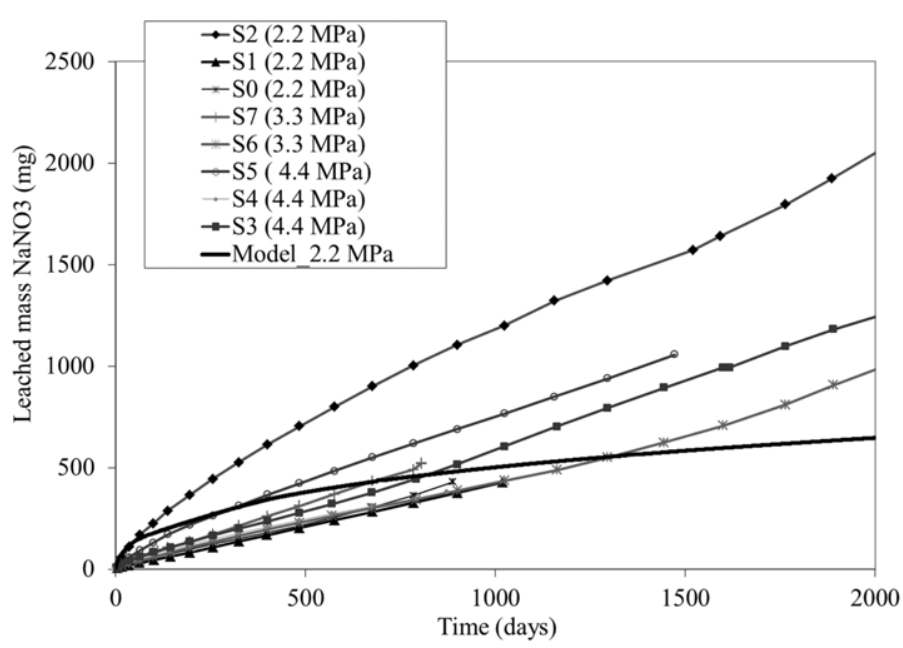

Fig. 9. Case 3. (a) Volumetric deformation v. time. (b) Leached mass of $\mathrm{NaNO}_{3}$. Experimental results and model predictions. 


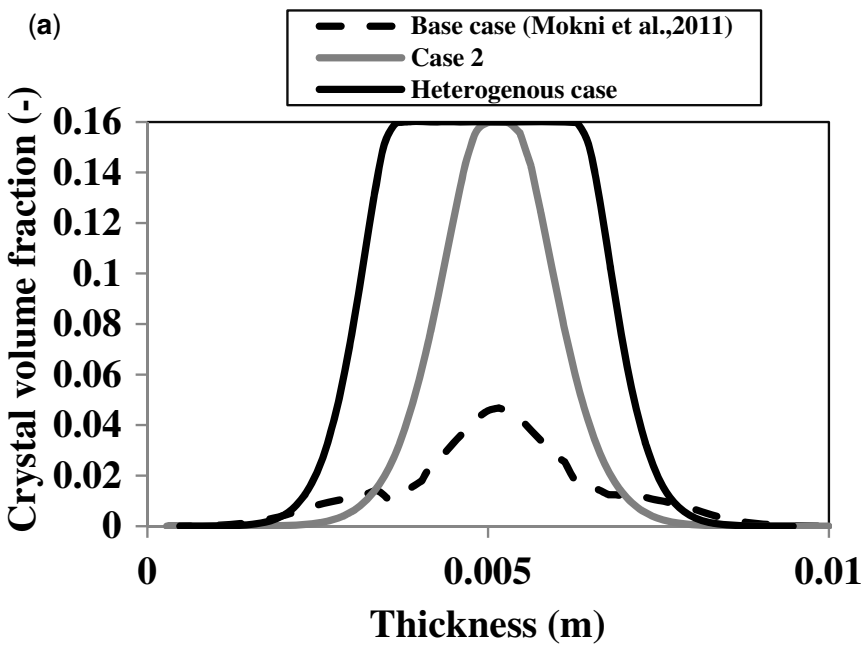

(b)

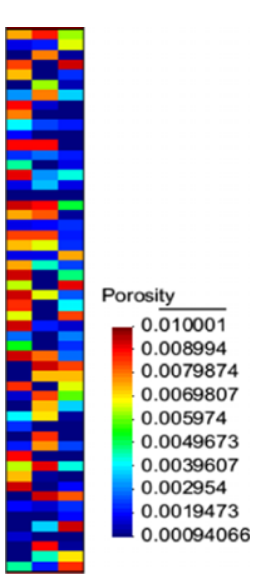

Fig. 10. (a) Profiles of crystal volume fraction after 1500 days of hydration for case 2, heterogeneous case and base case (Mokni et al. 2011). (b) Heterogeneous distribution of porosity (each finite element is characterized by a single value of porosity).

in contact with water the $\mathrm{NaNO}_{3}$ salt crystals at the outer boundary of the material will dissolve rapidly. This results in the formation of pores filled with a highly saturated $\mathrm{NaNO}_{3}$ solution. As a consequence permeability and diffusivity increase but most of the water that is taken up further by the BW stays in the first layer of pores to dissolve the remaining crystals and in a next strep to dilute the saturated $\mathrm{NaNO}_{3}$ solution. Profiles of crystal volume fraction (Fig. 10a) allow the thickness of the leached zones to be calculated. Table 3 summarizes the calculated and measured leached thicknesses (obtained by $\mu \mathrm{CT}$ and ESEM analyses; Valcke et al. 2010) at different times for the different analysed cases. For case 2 , the model predicts a slower progress of the water front in comparison to the base case presented in Mokni et al. (2011). However, even for this slower progress of the water front, there is still a difference between the modelled results and the $\mu \mathrm{CT}$ analyses, which show that after about $c .1500$ days of hydration the water front had not yet reached the centre of the sample (Table 3). For case 3, the model predicts a slower dissolution of the crystals in the centre of the sample and a slower progression of the hydration front compared with the base case and case 2 (Table 3). Indeed, except for the outermost layers, the largest part of the sample is not hydrated after 2000 days. This is a direct consequence of the size reduction of the outer pores, which induces a decrease in the permeability and the diffusion coefficient, and an increase of the osmotic efficiency in these compressed layers, while deeper in the sample these parameters are varying in the opposite way. This maintains a very slow inflow of water towards the sample and outflow of the solute over a long time.

\section{Analysis of the effect of heterogeneous distribution of porosity on leached thickness of $B W$ and hydration front}

Visual examination of the $\mu \mathrm{CT}$ images after different hydration periods shows irregular shape of

Table 3. Calculated and measured leached thickness (note that these data relate to the total thickness, that is, the sum of the thicknesses of the two leached layers per sample)

\begin{tabular}{lccccc}
\hline $\begin{array}{l}\text { Duration } \\
(\text { days })\end{array}$ & $\begin{array}{c}\text { Leached } \\
\text { thickness } \\
(\mathrm{mm})\end{array}$ & $\begin{array}{c}\text { Leached } \\
\text { thickness } \\
\text { calculated } \\
(\mathrm{mm})(\text { case } 2)\end{array}$ & $\begin{array}{c}\text { Leached } \\
\text { thickness } \\
\text { calculated } \\
(\mathrm{mm})(\text { case 3) }\end{array}$ & $\begin{array}{c}\text { Leached thickness } \\
\text { calculated }(\mathrm{mm}) \\
\text { (heterogenous } \\
\text { case) }\end{array}$ & $\begin{array}{c}\text { Leached thickness } \\
\text { calculated (base } \\
\text { case) }(\mathrm{mm})\end{array}$ \\
\hline c. 1500 & 6.29 & 9 & 6.4 & c. 6.62 & $*$ Mokni et al. 2011) \\
\hline
\end{tabular}

*Hydration front has already reached the centre of the sample. 


\section{N. MOKNI ET AL.}

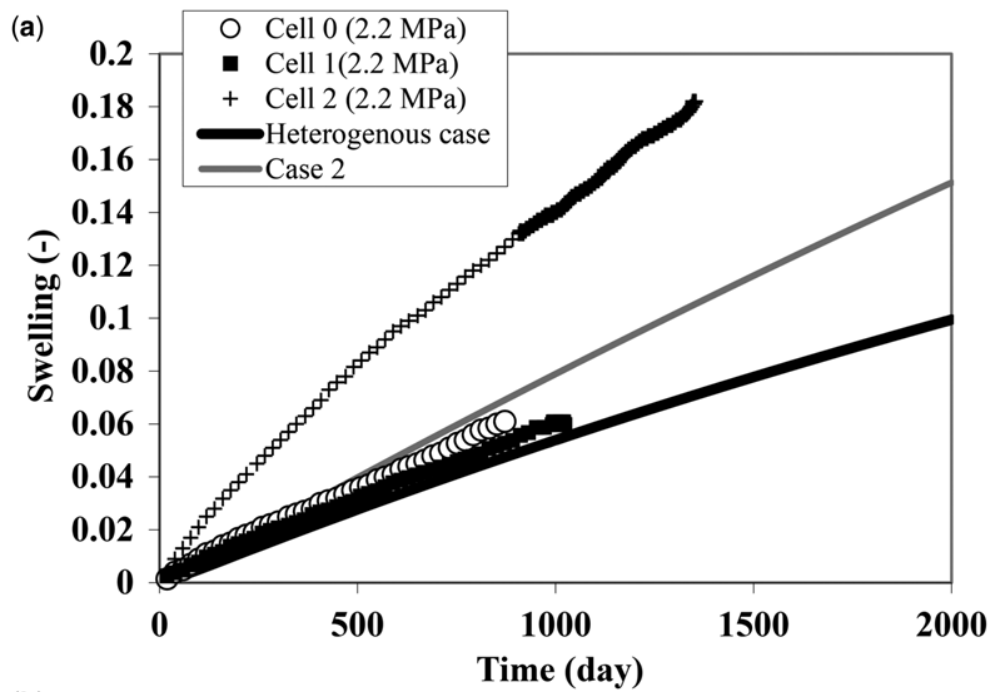

(b)

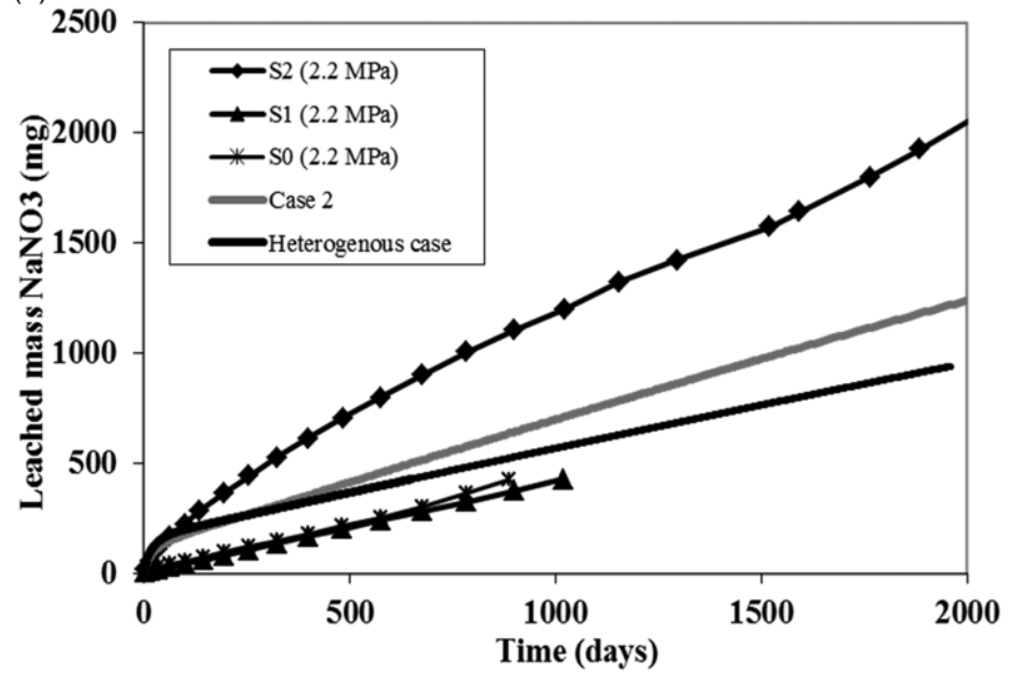

Fig. 11. Heterogeneous case. (a) Volumetric deformation v. time. (b) Leached mass of $\mathrm{NaNO}_{3}$. Experimental results and model predictions.

the hydration front (Fig. 1a), which can be attributed to pre-existing heterogeneities inside the BW leading to local variation of permeability, diffusivity and osmotic efficiency of the material. This heterogeneity may originate from the distribution of crystals, which are often grouped together in larger crystal clusters, generating zones with variable salt content and porosity (Fig. 1b).

In an additional effort to more realistically simulate the progression of the wetting front, some heterogeneity of the transport properties has been considered. The same transport function as in case 2 are used (Table 2, n=2) since they lead to good estimates of swelling and leaching rates. Figure 10b shows the initial porosity field considered. The mean porosity is $\phi_{\text {mean }}=0.01$ (same initial porosity as the homogenous case: case 2) and the variance is in the order of $10^{-5}$. This random field has no spatial correlation. The range of initial porosity considered is justified by the existence of only a few isolated pores with different sizes observed in ESEM images of dry BW (Valcke et al. 2010; Mariën et al. 2013). Note that, owing to the small variations in porosity considered, initial values of permeability, osmotic efficiency and diffusivity are different for each 
(a)

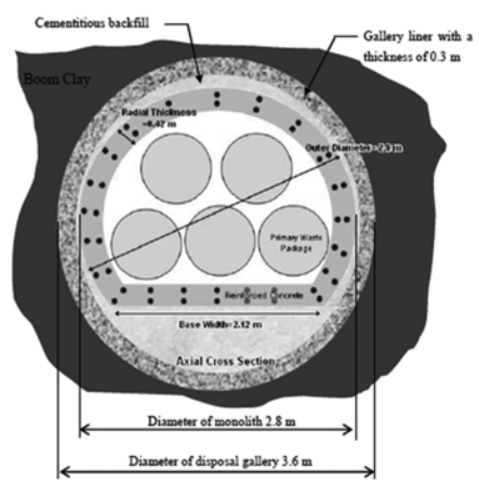

(b)

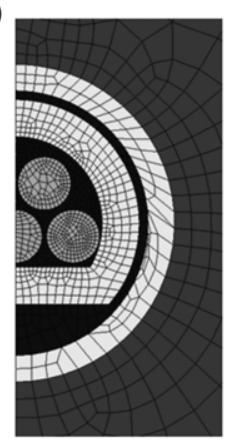

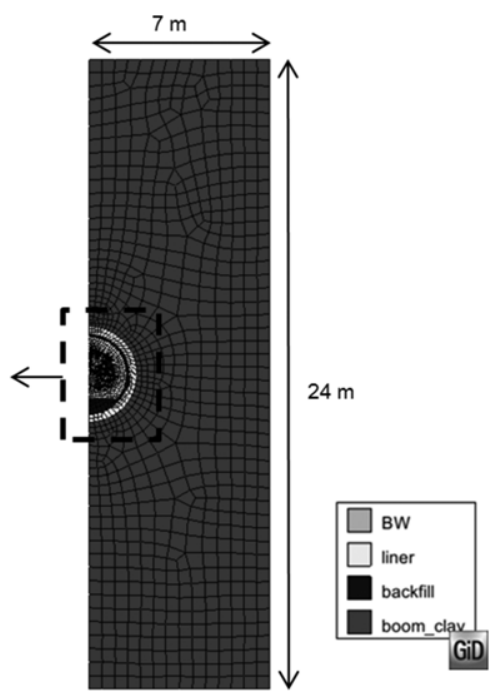

Fig. 12. (a) Axial cross section of the monolith for Eurobitum (Wacquier \& Van Humbeeck, 2009). (b) 2D Finite element model.

finite element. The profile of crystal volume fraction drawn after 1500 days for the heterogeneous case is shown in Figure 10a. When comparing the thickness of the hydrated layer with the experimental results, the heterogeneous case shows a comparable result (Table 3). Figure 11 shows the evolution of swelling and leached mass of $\mathrm{NaNO}_{3}$ with time. Although the model predicts a slower swelling and leaching rates as compared with case 2 , induced by the marked heterogeneity in the initial values of the transport properties, the results are still in the range of the experimental measurements.

\section{CH modelling of the in-situ disposal of BW}

According to the present disposal design of ONDRAF/NIRAS, ten 2201 drums, each filled with on the average 1701 of Eurobitum, would be grouped in two arrays of five drums in cement-based secondary containers, which are to be placed in concrete lined disposal galleries that are excavated at mid depth in the host formation (Fig. 12a; Wacquier \& Van Humbeeck 2009). The void space between the primary waste packages and the secondary concrete container, and between the secondary concrete container and the gallery liner, will

Table 4. Initial values of transport functions for the different materials considered in the in-situ case

\begin{tabular}{llll}
\hline Parameters (initial values) & & Symbols & Values \\
\hline BW & Crystal volume fraction & $\phi_{c 0}$ & 0.16 \\
& Porosity & $\phi f_{0}$ & 0.01 \\
& Intrinsic permeability & $k_{0}\left(\mathrm{~m}^{2}\right)$ & $\alpha \times 10^{-26}$ \\
& & $\beta$ & 1 \\
& & $\sigma_{0}$ & 0.95 \\
& Efficiency coefficient & $n$ & 2 \\
& & $D\left(\mathrm{~m}^{2} \mathrm{~s}^{-1}\right)$ & $1.6 \times 10^{-16}$ \\
Boom Clay & Diffusion coefficient & $\kappa\left(\mathrm{kg} \mathrm{s}^{-1} \mathrm{~m}^{-3}\right)$ & 0.47 \\
& Dissolution rate constant & $w_{l}^{s}$ & $10^{-19}$ \\
Concrete container+liner & Solute mass fraction & $K\left(\mathrm{~m}^{2}\right)$ & 0.001 \\
& Intrinsic permeability & $w_{l}^{s}$ & $10^{-18}$ \\
Backfill & Solute mass fraction & $K\left(\mathrm{~m}^{2}\right)$ & 0.001 \\
& Intrinsic permeability & $w_{l}^{s}$ & $10^{-16}$ \\
& Solute mass fraction & $K\left(\mathrm{~m}^{2}\right)$ & 0.001
\end{tabular}


(a)

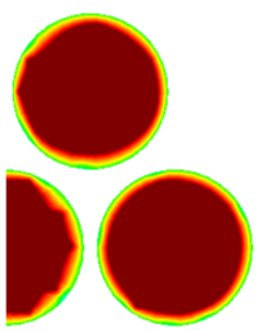

0.01 days

(b)

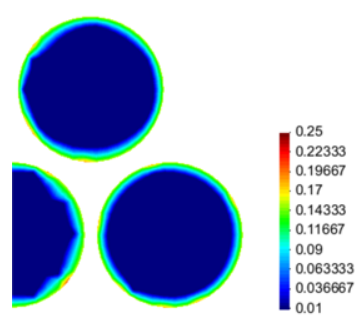

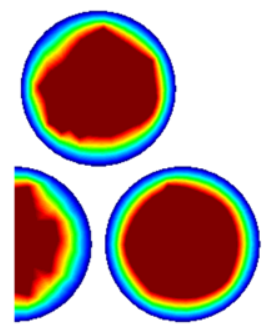

5 years

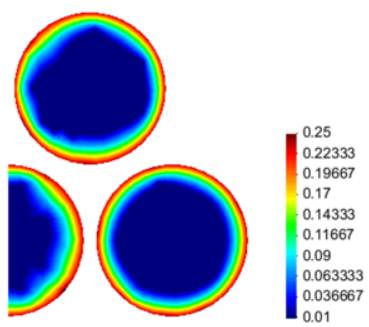

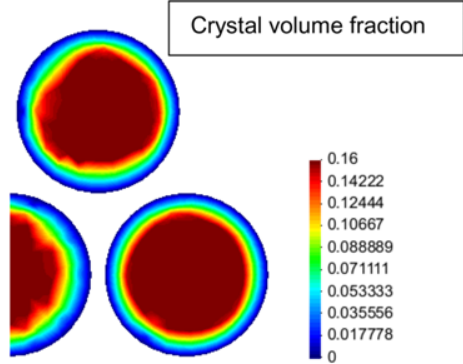

GiD

100 years

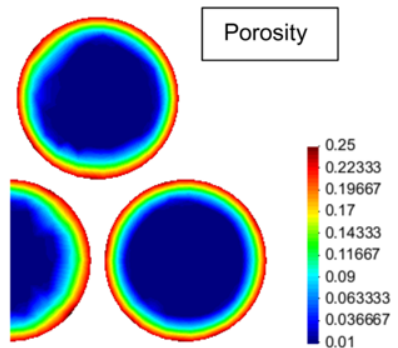

Fig. 13. Contours of crystal volume fraction (a) and porosity in BW drums (b) after 0.01 days, and 5 and 100 years

be backfilled with cement-based materials. It is important to recognize that several aspects of the Eurobitum disposal system are not yet finalized, in particular the composition of the concrete secondary container, the presence or the absence of reinforcement bars in the secondary concrete contained and the composition of the cement based backfill material. The design provided in Figure $12 \mathrm{a}$ is based on the latest information and assumptions.

In the frame of the study of the long-term behaviour of Eurobitum bituminized radioactive waste in final disposal conditions, a preliminary hydrochemical analysis is presented here. The objective is to obtain first insights into the kinetics of water uptake, dissolution, solute leaching and maximum generated swelling and pressure under disposal conditions. A 2D finite element model is used considering a section of the gallery taken perpendicularly to the axial direction (Fig. 12b). The cementitious backfill, the concrete container, the concrete liner and the Boom Clay are considered to be saturated with water with low $\mathrm{NaNO}_{3}$ concentration (initial mass fraction is $w=0.001 \mathrm{~kg} \mathrm{~kg}^{-1}$ ). The BW filling the drums contains $\mathrm{NaNO}_{3}$ crystals occupying $16 \%$ of the total volume of the BW (corresponding to $28 \%$ in weight) and is considered to

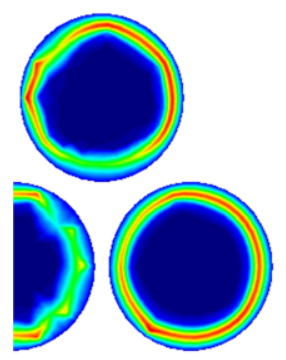

5 years

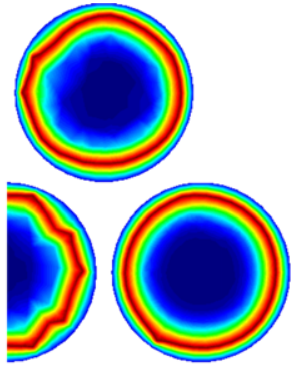

20 years

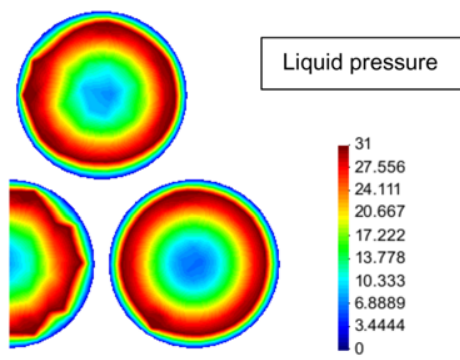

100 years

Fig. 14. Contour field of liquid pressure in BW drums after 5, 20 and 100 years. 
(a)

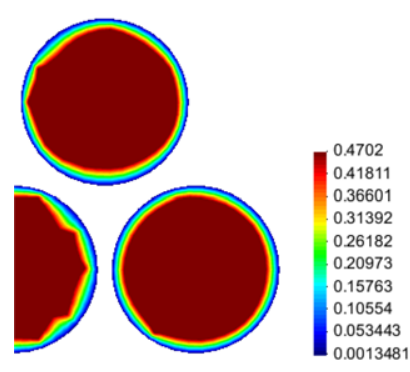

(b)

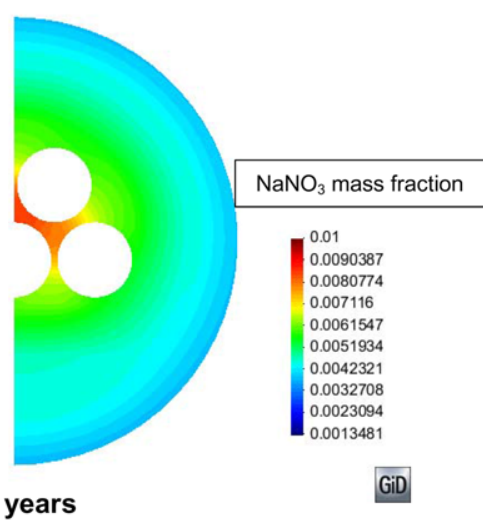

(c)

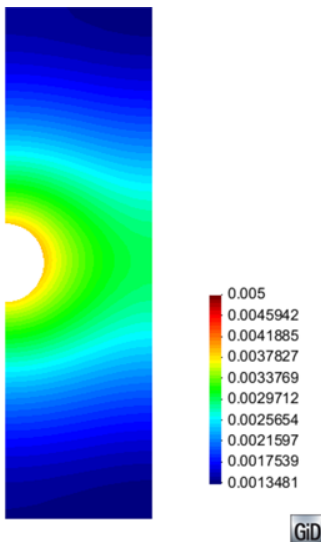

Fig. 15. Contour field of NaNO3 mass fraction in the liquid phase after 100 years in (a) BW, (b) backfill, concrete container and liner and (c) Boom Clay.

be saturated with $\mathrm{NaNO}_{3}$ brine (initial mass fraction: $w=0.47 \mathrm{~kg} \mathrm{~kg}^{-1}$ ). The same initial values for permeability, osmotic efficiency and diffusion coefficient for BW as for case 2 are used for this analysis. Detailed materials parameters are summarized in Table 4.

Figure 13 shows the contour field of crystal volume fraction after, 0.01 days, and 5 and 100 years together with contours of porosity. At early time, owing to high concentration gradient, water is attracted from the backfill to the BW. After 5 years, the crystal volume fraction $\left(\phi_{c}\right)$ decreases and tends to zero at the outer boundary of the drums, while no change in $\phi_{c}$ is observed in the middle of the drum after 100 years (Fig. 13a). This suggests that it will take several hundred years before the hydration front has reached the centre of the drums. As a consequence of crystal dissolution, porosity increases at the hydrated layers (Fig. 13b). No compression of the outer hydrated boundary is observed since no mechanical coupling is considered. Figure 14 shows contour field of liquid pressure after 5, 20 and 100 years. The figure shows that there is a pressure front that progresses slowly towards the centre of the drums. After 5 years the maximum value is attained at the outer boundary of the BW drums and is around $31 \mathrm{MPa}$. A decrease of the maximum value of liquid pressure is also observed after 100 year at the outer hydrated layers. In fact, the increase of porosity at this zone induces an increase of permeability and diffusion coefficient and a decrease of efficiency of the membrane. Drainage and solute diffusion out of the BW are favoured, resulting in a decrease in osmotic pressure and therefore in pore pressure. Figure 15a shows contours field of concentration after 100 years in BW. Except for the outer boundary where the crystals have dissolved, the concentration of the $\mathrm{NaNO}_{3}$ solution inside the $\mathrm{BW}$ drums remains constant and equal to that of a saturated $\mathrm{NaNO}_{3}$ solution after 100 years. In fact, the crystals provide a source term of solutes that prevent the decrease of the concentration. Contours field of concentration through the backfill material, liner, concrete container and Boom Clay after 100 years are shown in Figures $15 \mathrm{~b}$ and $\mathrm{c}$. Because the $\mathrm{BW}$ is not a perfect semipermeable membrane, the solute is allowed to diffuse out of BW and the $\mathrm{NaNO}_{3}$ concentration increases in the surrounding materials.

\section{Conclusion}

In this paper, equations of the key transport variables in the CHM formulation proposed by Mokni et al. (2011) are improved in order to better reproduce some of the experimental results. In this context a new expression of the osmotic efficiency coefficient $(\sigma)$, including the dependence of $\sigma$ on porosity and on crystal volume fraction, has been proposed. A new expression describing BW permeability evolution with porosity has been presented as well. The modelling results illustrate that $\alpha$ and $\beta$, parameters controlling the rate of change of intrinsic permeability with porosity, have a significant influence on swelling and leaching rates and on progression of the hydration front. The proposed functions are interdependent and control the magnitude of the coupled fluxes, namely chemical osmosis and ultrafiltration. In addition, it was demonstrated that assuming a simple realization of random fields of porosity that is of transport properties (permeability, osmotic efficiency and 


\section{N. MOKNI ET AL.}

diffusivity) improves the modelling results in terms of progression of the hydration front. Indeed, in this case, the calculated and the measured thicknesses of the leached layers, after 1500 days of hydration, were quite similar. This approach implies different magnitude of direct and coupled fluxes at each finite element in the domain. Accounting for spatial and temporal variations of the key transport parameters was essential to reproduce all the aspects that were observed in the experiments (swelling, leaching of sodium nitrate and thickness of the leached layer). Finally, the hydro-chemical analysis of the behaviour of Eurobitum drums under disposal conditions provided first insights into the kinetics of water uptake, dissolution, solute leaching and maximum generated pressure under disposal conditions.

This work was undertaken in close cooperation with, and with the financial support of ONDRAF/NIRAS, the Belgian Agency for the Management of Radioactive Waste and Enriched Fissile Materials, as part of its programme on geological disposal of medium-level long-lived waste.

\section{References}

Mariën, A., Mokni, N., Valcke, E., Olivella, S. Smets, S. \& LI, X. 2013. Osmosis-induced water uptake by Eurobitum bituminized radioactive waste and pressure development in constant volume conditions. Journal of Nuclear Materials, 432, 348-365.
MoKNI, N. 2011. Deformation and Flow Induced by Osmotic Processes in Porous Materials. PhD thesis, Universitat Politècnica de Catalunya, España.

Mokni, N., Olivella, S., Li, X., Smets, S. \& Valcke, E. 2008. Deformation induced by dissolution of salts in porous media. Physics and Chemistry Earth, Parts $A / B / C, \mathbf{3 3}$ (Supplement 1), S436-S443.

Mokni, N., Olivella, S. \& Alonso, E. E. $2010 a$. Swelling in clayey soils induced by the presence of salt crystals. Applied Clay Science, 47, 105-112.

Mokni, N., Olivella, S., Li, X., Smets, S., Valcke, E. \& MARIËN, A. 2010b. Deformation of bitumen based porous material: experimental and numerical analysis. Journal of Nuclear Materials, 404, 144-153.

Mokni, N., Olivella, S., Valcke, E., Mariën, A., SMETS, S. \& LI, X. 2011. Deformation and flow driven by osmotic processes in porous materials: application to bituminized waste materials. Transport in Porous Media, 86, 665-692.

Olivella, S., Gens, A., Carrera, J. \& Alonso, E. E. 1996. Numerical formulation for a simulator (CODE_BRIGHT) for the coupled analysis of saline media. Engineering with Computers, 13, 87-112.

ONDRAF/NIRAS 2009. The Long-term Safety Assessment Methodology for the Geological Disposal of Radioactive waste. ONDRAF/NIRAS report NIROND TR-2009-14 E, www.nirond.be

Valcke, E., Mariën, A., Smets, S., Li, X., Mokni, N. \& Olivella, S. 2010. Osmosis-induced swelling of Eurobitum bituminized radioactive waste in constant stress conditions. Journal of Nuclear Materials, 406, 304-316.

Wacquier, W. \& Van Humbeeck, H. 2009. B\&C Concepts and Open Questions. ONDRAF/NIRAS Technical note, 2009-0146. 